\title{
ОПИСАНИЕ УСТОЙЧИВОСТИ ГИДРОДИНАМИЧЕСКОЙ СИСТЕМЫ С ИСПОЛЬЗОВАНИЕМ ЧАСТОТНОГО МЕТОДА СИНТЕЗА МНОГОМЕРНЫХ СИСТЕМ НА ПРИМЕРЕ МЕСТОРОЖДЕНИЯ «ВЕРХНЕБЕРЕЗОВСКАЯ ПЛОЩАДЬ»
}

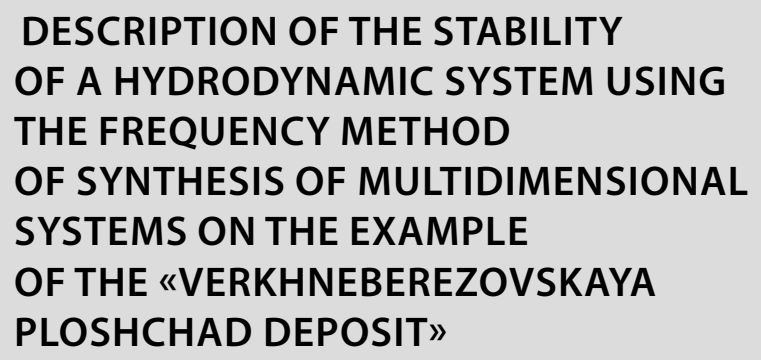

I. Kaliberda

Summary. The mathematical model of the mineral water deposit "Verkhneberezovskaya square" is considered. A hodograph is described in the form of logarithmic amplitude and phase frequency surfaces, which can be used in the interpretation of the Nyquist stability criterion by graphs. A frequency method for the synthesis of multidimensional systems is considered, when the input effects to a distributed controller are realized in the form of a discrete function over space. The condition under which the object belongs to the class of spatially invariant is shown. It is concluded that the frequency method of synthesis of regulators is a convenient tool for describing the stability of hydrodynamic systems.

Keywords: method of synthesis of regulators, control system with distributed parameters, hydrolithospheric processes.

\section{Ввецение}

$\Pi$ роцессы, происходящие в реальной среде, содержат как временную составляющую, так и координаты трехмерного пространства. Математические уравнения, описывающие модели рассматриваемых изменений, выражаются, как правило с использованием частных производных. Системный анализ таких изменений, в связи с этим, сильно усложняется.

В качестве объекта исследования выступает месторождение минеральных вод «Верхнеберезовская площадь», расположенное к югу от г. Кисловодска. Необходимо описать устойчивость гидродинамической системы. Так как в данном случае не существует

\author{
Калиберда Игорь Владимирович \\ Институт сервиса, туризма и дизайна (филиал) \\ СКФУ в г. Пятигорске \\ kaliberda.igor@ya.ru
}

Аннотация. Рассмотрена математическая модель месторождения минеральных вод «Верхнеберезовская площадь». Описан годограф в виде логарифмических амплитудной и фазовой частотных поверхностей, который можно использовать в интерпретации критерия устойчивости Найквиста по графикам. Рассмотрен частотный метод синтеза многомерных систем, когда входные воздействия в распределенный регулятор реализуются в виде дискретной функции по пространству. Показано условие, при котором объект принадлежит к классу пространственно-инвариантных. Сделан вывод, что частотный метод синтеза регуляторов представляется удобным инструментом при описании устойчивости гидродинамических систем.

Ключевые слова: метод синтеза регуляторов, система управления с распределенными параметрами, гидролитосферные процессы.

решения математической модели, применяется метод аппроксимации, который описывает действие объекта по выбранным пространственным модам. По сути предлагается дискретный аналог математической модели, который в свою очередь обладает численным решением. Наиболее известные методы данного направления:

- МКА - методы конечномерной аппроксимации;

- ДБСУ - декомпозиции билинейных систем управления;

- ЧМ - частотный метод.

МКА в литературе подробно рассмотрены, например, такими авторами как К.Г. Валеев и О. А. Жаутыков. В частности, для бесконечных систем ДУ даны теоремы существования решений и, причем их единственности. Развивая теорию управления и идентификации отно- 


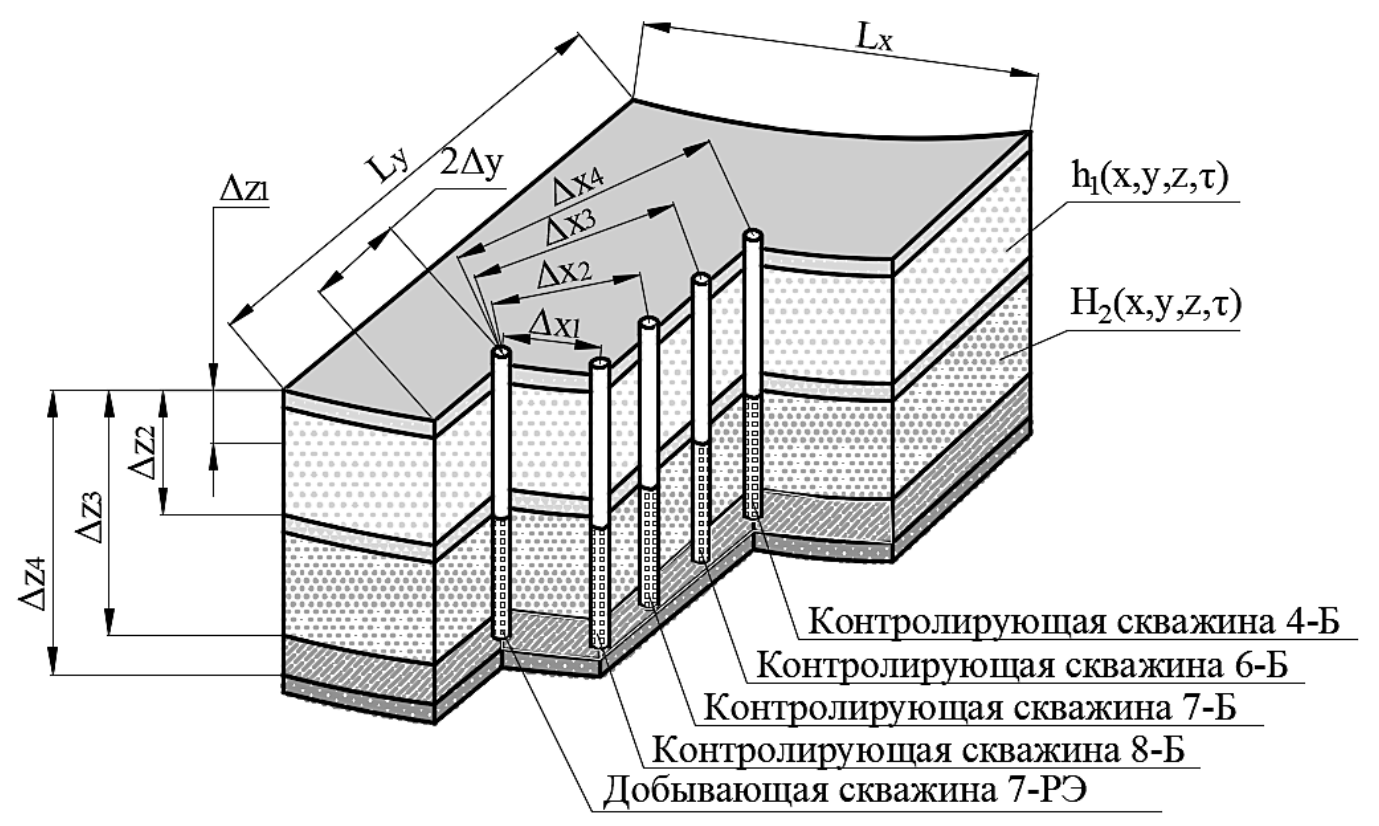

Рис. 1. Схема месторождения

Описание пород:

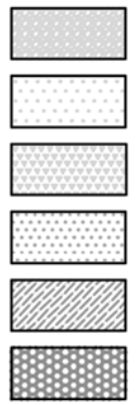

- валунно-галечниковые отложения;

- песчанник красновато-бурый кварцевый;

- гранитная дресва;

- песчаннык разноокрашенный, глинистый, слюдистый;

- глина красная песчанистая;

- кристаллические сланцы зеленовато-серые;

сительно билинейных динамических систем, Ю.И.Самойленко [9] разработал теоретико-групповые методы оптимизации и ДБСУ. Информационная энтропия протонной подсистемы была рассмотрена как носитель дискретной информации в описываемых молекулярных сенсорах и вычислительных устройствах, что и стало основой метода управления расположением протонов в водородных связях. Частотный метод (ЧМ) разработан И.М. Першиным и подробно изложен в работах [1-4]. ЧМ используется в системах с распределенными параметрами (СРП), когда для динамических характеристик объектов при выбранном числе пространственных мод определяются на основе экспериментальных данных. ЧМ СРП включает следующие составные части:

1. Методика анализа.

2. Определение частотных критерий устойчивости.

3. Аппроксимация характеристик объектов с учетом набора сосредоточенных звеньев.

4. Формирование структуры регулятора.

5. Методика синтеза для СРП.
Особое внимание уделяется переходу от бесконечно мерного фазового пространства к конечномерному. Если на данном этапе не учесть существенных свойств распределенного объекта, то в итоге возможно получить описание совсем другого процесса. Предпочтение решениям данного направления отдано частотному методу.

\section{Описание метола}

Рассмотрим математическую модель месторождения минеральных вод «Верхнеберезовская площадь», состоящую из одной добывающей скважины, четырех контролирующих скважин и двух водоносных пластов $\left(h_{1}, H_{2}\right)$. Схема расположения скважин представлена на рисунке 1.

Процесс взаимосвязей между пластами может быть записан в виде системы уравнений на стр. 50, где: 


$$
\left\{\begin{array}{l}
\frac{\partial h_{1}(x, y, z, \tau)}{\partial \tau}=k_{1, x} \frac{\partial^{2} h_{1}(x, y, z, \tau)}{\partial x^{2}}+k_{1, y} \frac{\partial^{2} h_{1}(x, y, z, \tau)}{\partial y^{2}}+k_{1, z} \frac{\partial^{2} h_{1}(x, y, z, \tau)}{\partial z_{1}^{2}} \\
\frac{\partial H_{2}(x, y, z, \tau)}{\partial \tau}=\frac{1}{\eta_{2}}\left(k_{2, x} \frac{\partial^{2} H_{2}(x, y, z, \tau)}{\partial x^{2}}+k_{2, y} \frac{\partial^{2} H_{2}(x, y, z, \tau)}{\partial y^{2}}+k_{2, z} \frac{\partial^{2} H_{2}(x, y, z, \tau)}{\partial z_{2}{ }^{2}}\right) \\
+V \cdot \delta\left(x_{0, j}, y_{0, j}, z_{0, j}\right) \\
0<x<L_{x} ; 0<y<L_{y} ; 0<z<L_{z_{4}}
\end{array}\right.
$$

$h_{l}$ - напор в горизонте грунтовых вод;

$\mathrm{H}_{2}$ - напор во 2-м водоносном горизонте;

$k_{i, x}, k_{i, y}, k_{i, z}-$ коэффициенты фильтрации по пространственным координатам в горизонте грунтовых вод $(i=1,2)$;

$\eta_{i}$ - упругоемкость $i$-го пласта $(i=2)$;

$V=Q \times K \phi$-понижение напора, вызванное воздействием добывающей скважиной ( $Q$ - дебит добывающей скважины, $K \phi-$ заданный коэффициент);

$\delta\left(x_{0, j}, y_{0, j}, z\right)$ - функция, равная единице, если $x=x_{0, j}$, $y=y_{0, j}, z_{1} \leq z \leq z_{2}$, и равная нулю в других случаях;

$x, y, z$ - пространственные координаты;

$\tau$ - время.

Граничные условия горизонта грунтовых вод и вторым пластом записываются в следующем виде:

$$
\begin{aligned}
& h_{1}\left(x, y, L_{z_{1}}, \tau\right)=h_{1}\left(x, y, L_{z_{1}}, \tau\right)+ \\
& +b_{1} \cdot\left(H_{2}(x, y, 0, \tau)-h_{1}\left(x, y, L_{z_{1}}, \tau\right)\right), \\
& H_{2}(x, y, 0, \tau)=H_{2}(x, y, 0, \tau)- \\
& -b_{1} \cdot\left(H_{2}(x, y, 0, \tau)-h_{1}\left(x, y, L_{z_{1}}, \tau\right)\right) .
\end{aligned}
$$

Для нижней границы второго пласта условие будет иметь вид:

$$
\partial H_{2}\left(x, y, L_{z_{2}}, \tau\right) / \partial z=0
$$

Граничные условия по бокам моделируемой области будут иметь вид:

$$
\begin{aligned}
& h_{1}(0, y, z, \tau)=h_{1,0} ; H_{2}(0, y, z, \tau)=H_{2,0} \\
& \partial h_{1}\left(L_{x}, y, z, \tau\right) / \partial x=0 ; \partial H_{2}\left(L_{x}, y, z, \tau\right) / \partial x=0
\end{aligned}
$$

где $h_{1.0}, H_{2.0}$ - начальные состояния грунтовых вод пластов.

\section{Решение}

Для определения динамических характеристик месторождения минеральных вод «Верхнеберезовская площадь» необходима дискретная модель объекта. Схема дискретизации представлена на рисунке 2.

Для грунтовых вод модель будет иметь вид:

$$
\begin{aligned}
& \frac{\Delta h_{1, \eta, \gamma, \xi}}{\Delta \tau}=k_{1, x} \frac{h_{1, \eta-1, \gamma, \xi}-2 \cdot h_{1, \eta, \gamma, \xi}+h_{1, \eta+1, \gamma, \xi}}{(\Delta x)^{2}}+ \\
& +k_{1, y} \frac{h_{1, \eta, \gamma-1, \xi}-2 \cdot h_{1, \eta, \gamma, \xi}+h_{1, \eta, \gamma+1, \xi}}{(\Delta y)^{2}}+ \\
& +k_{1, z} \frac{h_{1, \eta, \gamma, \xi-1}-2 \cdot h_{1, \eta, \gamma, \xi}+h_{1, \eta, \gamma, \xi+1}}{\left(\Delta z_{1}\right)^{2}} ; \\
& 2<\eta<N_{x}-1 ; 2<\gamma<N_{y}-1 ; 2<\xi<N_{z 1}-1
\end{aligned}
$$

где:

$N_{x}, N_{y}$ - число точек дискретизации по координатам $x$ и $y$ соответственно;

$N_{z i}$ - число точек дискретизации і-го пласта по координате $z(i=1,2)$;

$\eta, \gamma, \xi$ - пространственные координаты.

Для второго пласта модель будет иметь аналогичный вид.

Входным воздействием на объект управления служит дебит добывающей скважины $Q\left({ }_{\tau}\right)$. В работах [1-3] достаточно подробно описаны передаточные функции (ПФ) распределенных звеньев. Реакция объекта по выбранной моде $(\eta, \gamma)$ входного воздействия будет представлена в виде: 


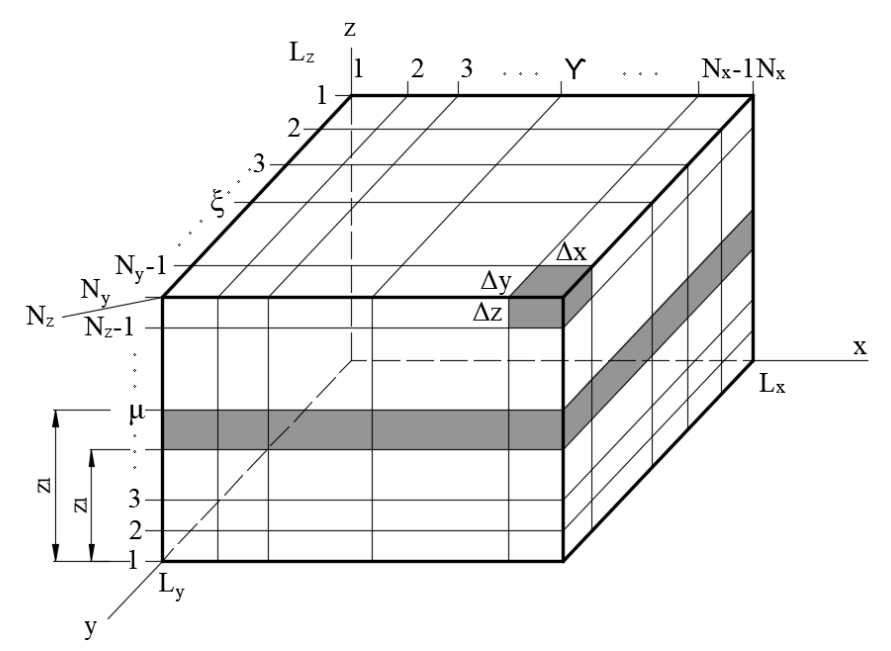

Рис. 2. Схема дискретизации

$W_{0, \eta, \gamma}(s)=\frac{\exp \left(\beta_{\eta, \gamma} \cdot z^{*}\right)+\exp \left(-\beta_{\eta, \gamma} \cdot z^{*}\right)}{\lambda \cdot \beta_{\eta, \gamma} \cdot\left(\exp \left(\beta_{\eta, \gamma} \cdot z_{L}\right)-\exp \left(-\beta_{\eta, \gamma} \cdot z_{L}\right)\right)}$,

$(\eta, \gamma=\overline{1, \infty})$

где:

$\beta_{\eta, \gamma}=\left(\frac{s}{a}+\psi_{\eta}^{2}+\widetilde{\varphi}_{\gamma}^{2}\right)^{1 / 2}$

$s$ - оператор Лапласа;

$*$

a, z, $z_{L}$ - заданные числа;

$\psi_{\eta}, \tilde{\varphi}_{\gamma}$ - пространственные частоты; $(\eta, \gamma=\overline{1, \infty})$.

Теперь объект с СРП можно описать в виде ПФ полученного образца (1), а каждая ПФ - отношением бесконечных полиномов.

Представим в (1) $s=j \omega$ и получим комплексный передаточный коэффициент (КПК) СРП по пространственным модам:

$$
\begin{aligned}
& W_{0, \eta, \gamma}(j \omega)=\frac{\exp \left(\beta_{\eta, \gamma} \cdot z^{*}\right)+\exp \left(-\beta_{\eta, \gamma} \cdot z^{*}\right)}{\lambda \cdot \beta_{\eta, \gamma} \cdot\left(\exp \left(\beta_{\eta, \gamma} \cdot z_{L}\right)-\exp \left(-\beta_{\eta, \gamma} \cdot z_{L}\right)\right)} \\
& (\eta, \gamma=\overline{1, \infty})
\end{aligned}
$$

где:

$\beta_{\eta, \gamma}=\left(\frac{j \omega}{a}+\psi_{\eta}^{2}+\tilde{\phi}_{\gamma}^{2}\right)^{1 / 2}$
СРП, у которой КПК можно описать выражением (2), относится к классу пространственно-инвариантных. Из этого следует, что решение при квазистационном воздействии распадается по собственным вектор-функциям оператора объекта. Данные вектор-функции можно представить в виде комбинации $\sin (). \& \cos ($.$) . Теперь$ данный объект можно представить в виде совокупности независимых блоков с комплексными передаточными коэффициентами вида (2). Отсюда следует, что пространственная мода, при прохождении через объект управления, изменяет только амплитуду.

Если изменять в (2) $\omega$ от 0 до $\infty$, то получим бесконечный спектр частотных характеристик СРП, представленный на рисунке 3 , a.

Введем дискретную функцию (ДФ) $G \eta, \gamma$ и обозначим ее через выражение:

$$
G_{\eta, \gamma}=\psi_{\eta}^{2}+\tilde{\phi}_{\gamma}^{2},(\eta, \gamma=\overline{1, \infty})
$$

Данная функция изменяется в пределах $G_{i}=G_{1.1} \leq G_{\eta, \gamma} \leq \infty$. При переходе от ДФ $G_{\eta, \gamma}$ к непрерывной $G$, охватывающей весь спектр дискретных значений ДФ $G_{\eta, \gamma}$ выражение (1) примет вид:

$$
\begin{aligned}
& W_{0}(G, s)=\frac{\exp \left(\beta_{\eta, \gamma} \cdot z^{*}\right)+\exp \left(-\beta_{\eta, \gamma} \cdot z^{*}\right)}{\lambda \cdot \beta_{\eta, \gamma} \cdot\left(\exp \left(\beta_{\eta, \gamma} \cdot z_{L}\right)-\exp \left(-\beta_{\eta, \gamma} \cdot z_{L}\right)\right)}, \\
& (\eta, \gamma=\overline{1, \infty})
\end{aligned}
$$

где:

$\beta_{\eta, \gamma}=\left(\frac{s}{a}+G\right)^{1 / 2}, G_{i} \leq G \leq \infty$ 
a)
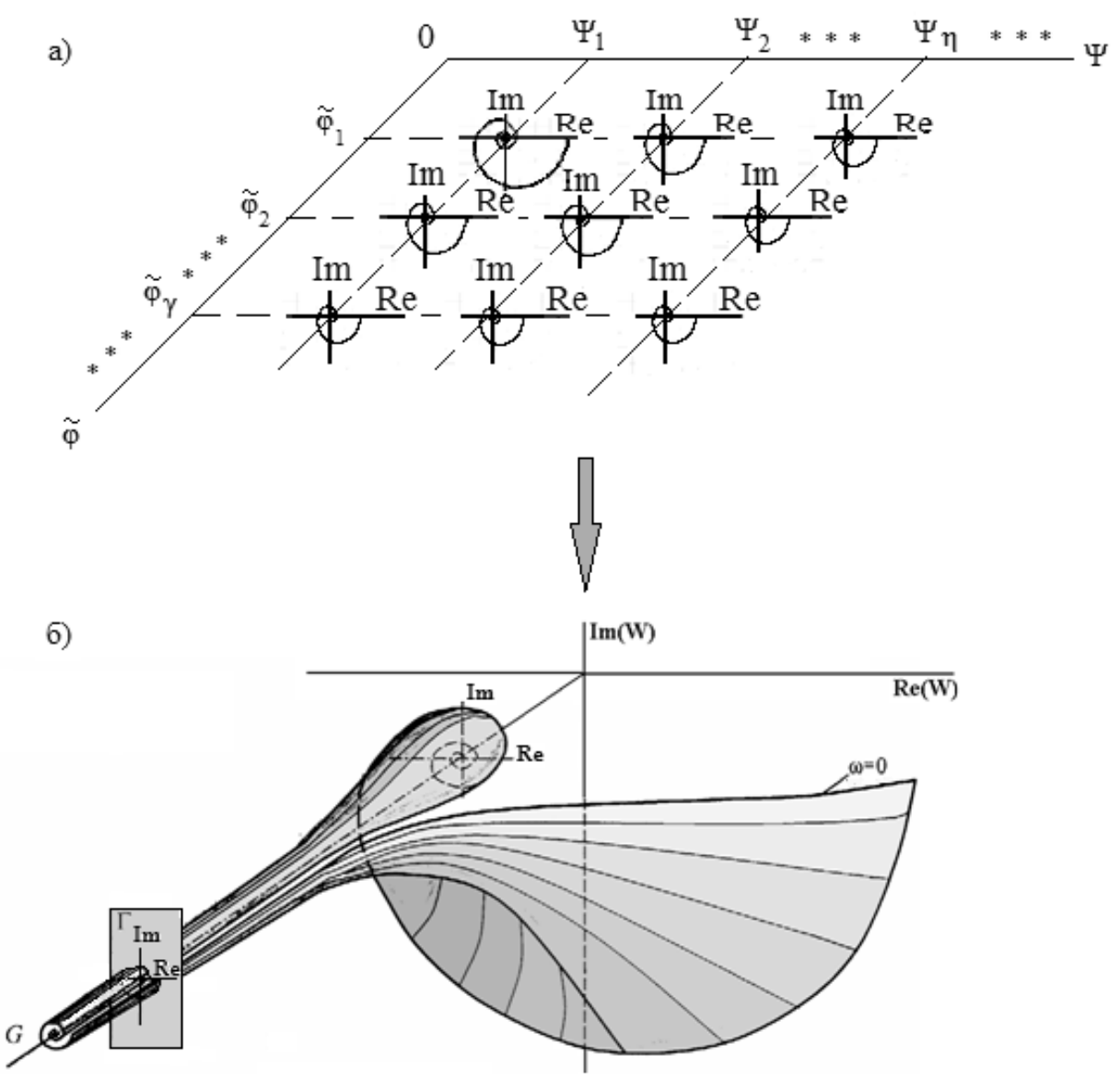

Рис. 3. Пространственный годограф
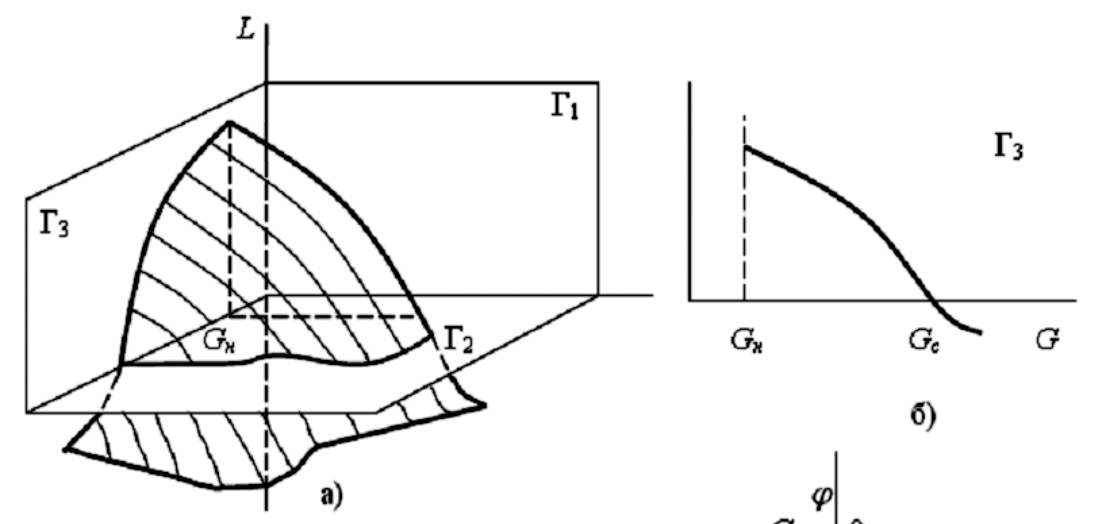

б)

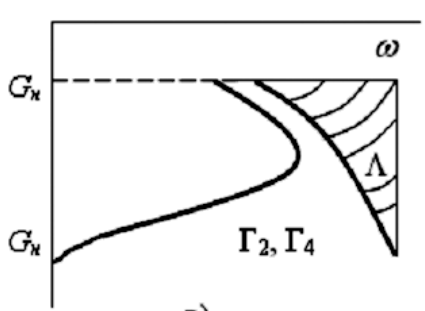

B)

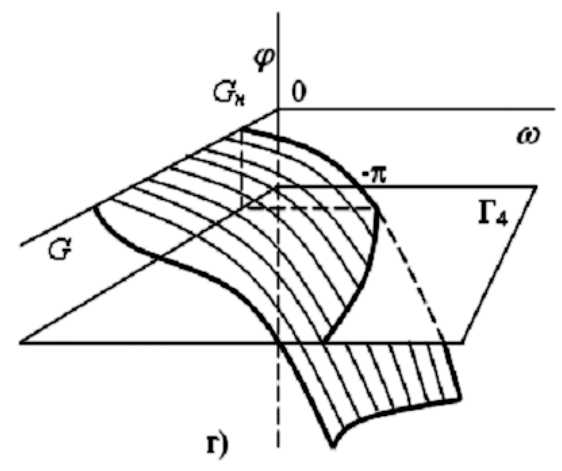

Рис. 4. Частотные поверхности 
В случаях гидродинамических систем, когда возможно оценить динамику процесса, передаточную функцию можно аппроксимировать функцией следующего вида:

$$
W(s, G)=K \cdot e^{-\beta \cdot \Delta z}, G_{i} \leq G \leq \infty,
$$

где:

$K, \Delta_{z}$ - параметры гидролитосферного объекта.

Изменяя в (4) $\omega$ от 0 до $\infty$, а $G_{i} \leq G \leq \infty$, получим пространственных годограф, включающий весь спектр частотных характеристик по пространственным модам, см. рис. 3, 6 .

Полученный годограф в виде логарифмических амплитудной и фазовой частотных поверхностей можно использовать для анализа устойчивости гидродинамической системы на основе критерия Найквиста по графикам. Положим, что характеристические полиномы рассматриваемой СРП по каждой пространственной моде не имеют полюсов, лежащих в правой полуплоскости. Тогда, критерием устойчивости замкнутой системы будет условие не пересечения линии среза модуля на совмещенной плоскости $\Gamma_{2}$ и $\Gamma_{4}$ с областью $A$, как показано на рисунке 4, в. На рисунке 4, а представлена амплитудная частотная поверхность, на рисунке 4, гфазовая поверхность.

Рассмотрим частотный метод синтеза многомерных систем. Входные воздействия в распределенный регулятор реализуются в виде дискретной функции по пространству. Значения функции выхода распределенного объекта измеряются в конечном числе точек. Матрица комплексных передаточных коэффициентов объекта, связывающая $\xi$-й вход с $m$-м выходом записывается в виде [1, 2]:

$$
W_{0, \eta, \gamma}(j \omega)=\left[W_{m, \xi}\right],(m, \xi=\overline{1, n}) .
$$

Представим входное воздействие на объект в виде ряда:

$$
\begin{aligned}
& \alpha_{\gamma}(\tau)=\sum_{\eta=1}^{n} \tilde{N}_{\eta}(\tau) \cdot \sin \left(\frac{\pi \cdot \eta \cdot y_{\gamma}}{L}\right) \\
& L=\Delta y \times(n+1) ; y_{\gamma}=\Delta y \times \gamma,(y=\overline{1, n})
\end{aligned}
$$

где: $\Delta y$ - заданное положительное число;

$y_{\gamma}$ - точки дискретизации $(\gamma=\overline{1, n})$.

Полагая в (6) $\tilde{N}_{\eta}(\tau)=\exp (j \omega \tau)$, где $\omega-$ круговая частота, определим реакцию объекта (6) на каждую пространственную моду:

$$
\begin{aligned}
& T_{\eta, \gamma}(j \omega \tau)=\exp (j \omega \tau) \cdot \sum_{\xi=1}^{n} W_{\gamma, \xi} \cdot \sin \left(\frac{\pi \cdot \eta \cdot \xi}{n+1}\right) \\
& (\eta, \gamma=\overline{1, n})
\end{aligned}
$$

Запишем комплексный передаточный коэффициент по каждой пространственной моде:

$$
W_{\eta}\left(y_{\gamma, j} \omega\right)=\frac{T_{\eta, \gamma}}{\exp (j \omega \tau) \cdot \sin (\pi \cdot \eta \cdot \gamma) /(n+1)}
$$

Объект принадлежит к классу пространственно-инвариантных, если (9) записывается в виде:

$$
W_{\eta}\left(y_{\gamma, j} \omega\right)=W_{\eta}(j \omega),(\gamma=\overline{1, n})
$$

Подставляя (9) в (10) и преобразуя, получим дискретный аналог условия пространственной инвариантности объекта [1, 2]:

$$
\sum_{\xi=1}^{n} W_{\gamma, \xi} \cdot \sin \left(\frac{\pi \cdot \eta \cdot \xi}{n+1}\right)=W_{\eta} \cdot \sin \left(\frac{\pi \cdot \eta \cdot \gamma}{n+1}\right)
$$

Запишем уравнение (11) в виде:

$$
\begin{aligned}
& W \cdot \chi=W_{\eta} \cdot \chi_{\eta} ; \chi_{\eta}=\left[\chi_{\eta, \xi}\right] \\
& \chi_{\eta, \xi}=\sin (\pi \cdot \eta \cdot \xi /(n+1)) \\
& (\xi=\overline{1, n}) ;(\gamma=\overline{1, n})
\end{aligned}
$$

Из соотношения (12) следует [1]: объект (6) принадлежит к классу пространственно-инвариантных, если $\chi_{\eta},(\eta=\overline{1, n})$ являются собственными векторами матрицы $W$.

\section{Зак^ючение}

Таким образом, ЧМ синтеза регуляторов представляется наиболее удобным инструментом при создании СРП, систем управления рассматриваемым процессом. В отличие от сосредоточенных систем частотный метод анализа и синтеза распределенных систем оперирует бесконечным набором пространственных мод (собственных вектор-функций оператора объекта). Состояние каждой пространственной моды описывается в бесконечном фазовом пространстве. Показано, что частотные характеристики распределенных объектов могут быть представлены бесконечной совокупностью частотных характеристик по пространственным модам. Поскольку рассматриваемые моды обладают свойствами ортогональности, то использование обобщенной координаты позволило перейти к амплитудным и фазовым частотным поверхностям. С помощью рассматри- 
ваемых частотных поверхностей возможно получить графическую интерпретацию критерия устойчивости Найквиста для выделенного класса систем с распределёнными параметрами на примере месторождения минеральных вод «Верхнеберезовская площадь». В современных работах авторов [4-5] разработаны специальные наборы звеньев и блоков, используемых в процедуре синтеза СРП управления.

\section{ЛИТЕРАТУРА}

1. Першин И. М. Анализ и синтез систем с распределенными параметрами. Издательство РИА КМВ. 2007. 243с.

2. Малков А.В., Першин И. М. Системы с распределенными параметрами. Анализ и синтез.— М.: Научный мир, 2012.—476с.

3. Першин И. М. Синтез систем с распределенными параметрами: проблемы и перспективы // Мехатроника, автоматизация, управление. 2005 . № 6. C. 2-10.

4. Першин И. М. Частотная концепция анализа и синтеза систем с распределенными параметрами: монография. — Пятигорск: ПФ СКФу. 2021. - 172 с.

5. Чернышев А.Б., Ильюшин Ю. В. Устойчивость распределенных систем с дискретными управляющими воздействиями. //Известия Южного федерального университета. — 2010.— № 12.—C. 166-171.

6. Рапопорт Э. Я. Оптимальное управление системами с распределенными параметрами. /Учебн. пособие.- М.: Высшая школа, 2009.

7. Хацкевич В.П. 0 решении задачи аналитического конструирования регуляторов для распределенных систем. //Автоматика и телемеханика.1972. 一 № 3.- С. 5-14.

8. Бутковский А.Г., Дарнинский Ю. В., Пустыльников Л. М. Управление распределенными системами путем перемещения источника. //Автоматика и телемеханика. - 1974. — № 5.- С. 11-30.

9. Бутковский А.Г., Самойленко Ю. И. Управление квантово-механическими процессами. — М.: Наука, 1984. — 256 с.

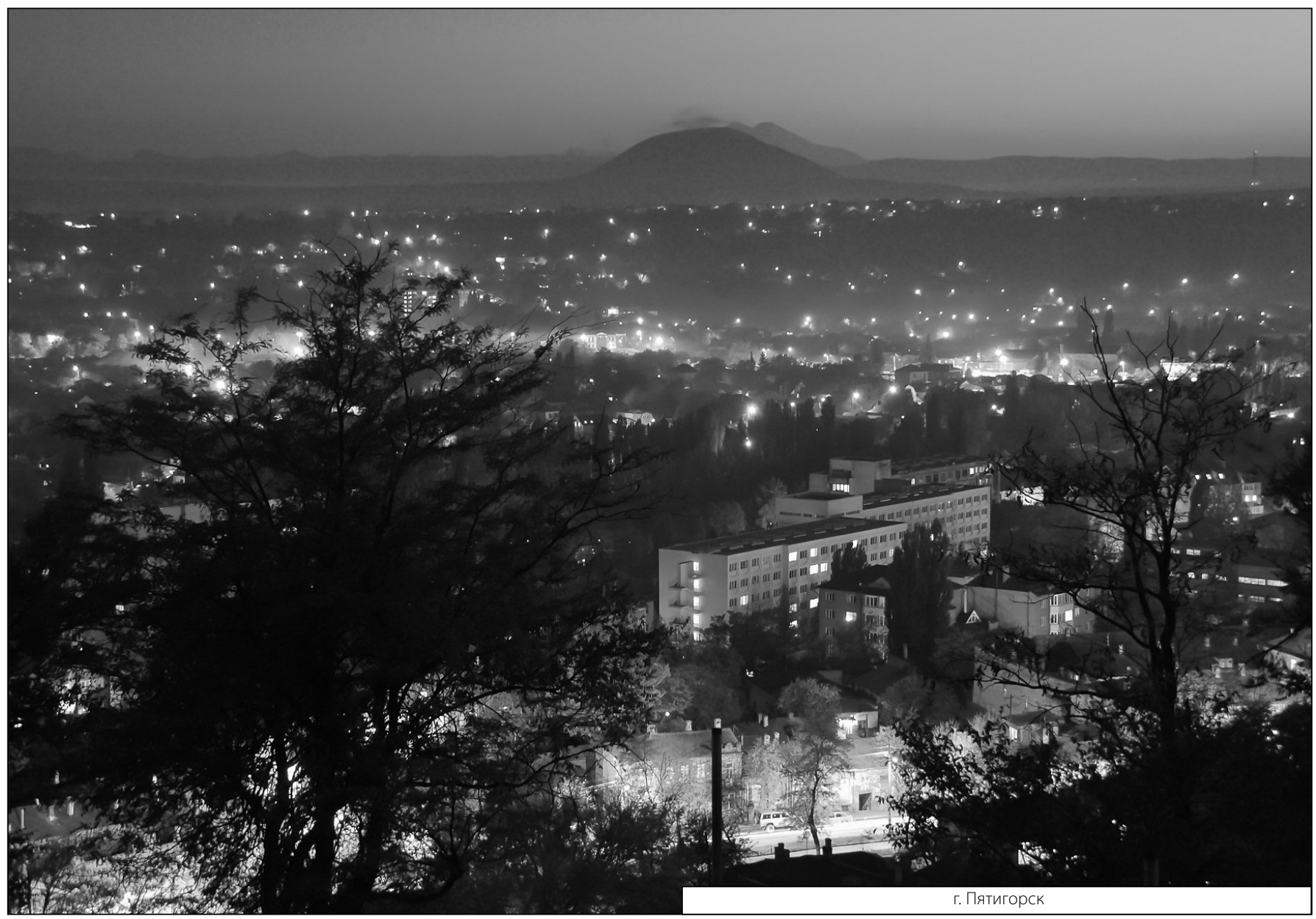

